\title{
Tail Asymptotic of Weibull-Type Risks
}

\author{
Enkelejd Hashorva and Zhichao Weng 1 \\ Department of Actuarial Science, University of Lausanne, Switzerland
}

August 22, 2018

\begin{abstract}
With motivation from Arendarczyk and Dȩbicki (2011), in this paper we derive the tail asymptotics of the product of two dependent Weibull-type risks, which is of interest in various statistical and applied probability problems. Our results extend some recent findings of Schlueter and Fischer (2012) and Bose et al. (2012).
\end{abstract}

Key Words: Weibull-type risks; FGM distribution; Gumbel max-domain of attraction; Supremum of Brownian motion; Elliptical distribution.

\section{Introduction}

In numerous statistical and probabilistic models various quantities of interest are defined in terms of product of random variables (or risks). For instance, given $X_{1}, X_{2}$ two positive risks, the product $Z=X_{1} X_{2}$ can be used to model a random deflation/inflation effect, if say $X_{1}$ is the deflator/inflator and $X_{2}$ is some base risk related to some financial loss. Since often the distribution functions of the risks are not known, the main interest is on the asymptotic analysis of the tail of $Z$. When $X_{1}$ is a bounded random variable, then $Z$ can be seen as a random contraction of $X_{2}$, see e.g., Berman (1992), Cline and Samorodnitsky (1994), Pakes and Navarro (2007), Hashorva and Pakes (2010), Hashorva et al. (2010,2012), Hashorva (2011,2012), Yang and Wang (2012). Interesting models where $X_{1}$ is unbounded have been studied in Cline and Samorodnitsky (1994), Maulik and Resnick (2004), Nadarajah (2005), Nadarajah and Kotz (2005), Zwart et al. (2005), Jessen and Mikosch (2006), Tang (2006a,b,2008), Liu and Tang (2010), Arendarczyk and Dębicki (2011,2012), Balakrishnan and Hashorva (2011), Chen (2011), Constantinescu et al. (2011), Jiang and Tang (2011), Yang et al. (2011), Schlueter and Fischer (2012) among several others.

With motivation from Arendarczyk and Dȩbicki (2011), in this paper we are concerned with the investigation of the tail asymptotics of the product $Z=X_{1} X_{2}$ of risks with Weibull tail behaviour i.e., for $X_{1}$ and $X_{2}$ such that

$$
\mathbb{P}\left\{X_{i}>x\right\} \sim g_{i}(x) \exp \left(-L_{i} x^{p_{i}}\right)
$$

with $g_{i}(\cdot)$ some regularly varying function at infinity and $L_{i}, p_{i}, i=1,2$ positive constants. In our notation $a(x) \sim$ $b(x)$, for two functions $a(\cdot)$ and $b(\cdot)$, means that $\lim _{x \rightarrow \infty} a(x) / b(x)=1$.

A large class of such risks satisfy (1.1) with $g_{i}(\cdot)$ a polynomial function i.e.,

$$
\mathbb{P}\left\{X_{i}>x\right\} \sim C_{i} x^{\alpha_{i}} \exp \left(-L_{i} x^{p_{i}}\right)
$$

with $C_{i}, p_{i}, L_{i}, i=1,2$ positive constants, $\alpha_{1}, \alpha_{2} \in \mathbb{R}$. A remarkable result of Arendarczyk and Dębicki (2011), which is crucial for the analysis of the extremes of Gaussian processes over random intervals, shows that when (1.2) 
holds, then

$$
\mathbb{P}\{Z>x\} \sim\left(\frac{2 \pi p_{2} L_{2}}{p_{1}+p_{2}}\right)^{\frac{1}{2}} C_{1} C_{2} A^{\frac{p_{2}}{2}+\alpha_{2}-\alpha_{1}} x^{\frac{2 p_{2} \alpha_{1}+2 p_{1} \alpha_{2}+p_{1} p_{2}}{2\left(p_{1}+p_{2}\right)}} \exp \left(-B x^{\frac{p_{1} p_{2}}{p_{1}+p_{2}}}\right)
$$

with

$$
A=\left[\left(p_{1} L_{1}\right) /\left(p_{2} L_{2}\right)\right]^{1 /\left(p_{1}+p_{2}\right)} \text { and } B=L_{1} A^{-p_{1}}+L_{2} A^{p_{2}} \text {. }
$$

Clearly, also $Z$ is Weibull-type risk, and thus (1.3) shows the closure property for the product of such risks.

The main goal of this paper is to investigate the tail asymptotics of $Z$ for Weibull-type risks allowing further for the risks to be dependent. In various theoretical problems and applications independence assumption is not tenable. Particular examples of the dependence structure assumed in this paper are risks with bivariate FairlyGumbel-Morgenstern (FGM) distribution. We also show by considering the special case that $X_{1}$ and $X_{2}$ are jointly Gaussian, that the dependence structure is crucial for the tail asymptotic of $Z$.

Our findings are of interest in various probabilistic models, for instance our Corollary 2.2 subsumes Theorem 1 in Bose et al. (2012) which is crucial for dealing with the spectral radius of random k-circulants; in particular that result implies the closure property of independent Weibull-type risks with respect to product. Our first application deals with the supremum of Brownian motion over random time interval. In the second application we extend the findings of Schlueter and Fischer (2012) which concern the calculation of the weak tail dependence coefficient of elliptical generalized hyperbolic distribution.

Outline of the rest of the paper: Section 2 presents the main findings of this contribution. In Section 3 we give two applications, followed by Section 4 where all the proofs are displayed.

\section{Main Results}

In this section both risks $X_{1} \sim F_{1}$ and $X_{2} \sim F_{2}$ are positive and satisfy (1.1) with $p_{i}, L_{i}$ positive constants, and $g_{i}$ regularly varying at infinity with index $\alpha_{i}, i=1,2$. Their dependence structure is modeled by a tractable conditions, namely we shall assume that for some positive measurable function $c(\cdot, \cdot)$ and some constants $K_{1}>0$, $K_{2}>0, \beta_{1}, \beta_{2} \in \mathbb{R}$

$$
\mathbb{P}\left\{X_{1}>x / y \mid X_{2}=y\right\}=\mathbb{P}\left\{X_{1}>x / y\right\} c(x, y) \quad \text { and } \quad K_{1} x^{\beta_{1}} \leq c(x, y) \leq K_{2} x^{\beta_{2}}
$$

are satisfied for all $x$ large and any $y>0$ and further

$$
\lim _{x \rightarrow \infty} \sup _{y \in\left[a_{1} w_{x}, a_{2} w_{x}\right]}\left|c(x, y)-D x^{q_{1}} y^{q_{2}-q_{1}}\right|=0
$$

holds for some constants $D>0,0<a_{1}<a_{2}, q_{1}, q_{2} \in \mathbb{R}$ and $w_{x}=x^{\frac{p_{1}}{p_{1}+p_{2}}}$.

Theorem 2.1. Let $X_{1}$ and $X_{2}$ be two dependent risks as above such that both $g_{1}, g_{2}$ are ultimately monotone. If condition (2.1) and (2.2) hold, then

$$
\mathbb{P}\{Z>x\} \sim D\left(\frac{2 \pi p_{2} L_{2}}{p_{1}+p_{2}}\right)^{\frac{1}{2}} A^{\frac{p_{2}}{2}+q_{2}-q_{1}} x^{\frac{2 p_{2} q_{1}+2 p_{1} q_{2}+p_{1} p_{2}}{2\left(p_{1}+p_{2}\right)}} g_{1}\left(z_{x}^{-1} x\right) g_{2}\left(z_{x}\right) \exp \left(-B x^{\frac{p_{1} p_{2}}{p_{1}+p_{2}}}\right),
$$

with $z_{x}=A x^{p_{1} /\left(p_{1}+p_{2}\right)}$ and $A$ and $B$ given by (1.4). 
Corollary 2.2. Under the conditions of Theorem [2.1, and $X_{1}, X_{2}$ are independence, then

$$
\mathbb{P}\{Z>x\} \sim\left(\frac{2 \pi p_{2} L_{2}}{p_{1}+p_{2}}\right)^{\frac{1}{2}} A^{\frac{p_{2}}{2}} x^{\frac{p_{1} p_{2}}{2\left(p_{1}+p_{2}\right)}} g_{1}\left(z_{x}^{-1} x\right) g_{2}\left(z_{x}\right) \exp \left(-B x^{\frac{p_{1} p_{2}}{p_{1}+p_{2}}}\right) .
$$

If additionally $X_{1}$ possess a positive pdf $h_{1}$ which is bounded and ultimately decreasing, then the pdf $h$ of $Z$ satisfies

$$
h(x) \sim L_{1} p_{1} A^{-p_{1}} x^{\frac{p_{1} p_{2}}{p_{1}+p_{2}}-1} \mathbb{P}\{Z>x\},
$$

provided that $h_{1}(x)=(1+o(1)) L_{1} p_{1} x^{p_{1}-1} \mathbb{P}\left\{X_{1}>x\right\}$.

Bose et al. (2012) derived in their Theorem 1 the tail asymptotics of the product of $n$ independent unit exponential random variables. The above corollary extends Theorem 1 of the aforementioned paper to the product of independent Weibull-type risks with common parameters $L$ and $p$ and $g$ being ultimately monotone. In fact, if $X_{i} \sim F, i=$ $1, \cdots, m$ are independent positive random variables such that (1.2) holds with $C, p, L$ positive constants, $\alpha \in \mathbb{R}$, then Theorem 1 of the aforementioned paper can be generalised to the following statement

$$
\mathbb{P}\left\{\prod_{i=1}^{m} X_{i}>x\right\} \sim m^{-\frac{1}{2}}(2 \pi L)^{\frac{m-1}{2}} C^{m} x^{\frac{2 m \alpha+(m-1) p}{2 m}} \exp \left(-m L x^{\frac{p}{m}}\right),
$$

which is a direct implication of the result of (1.3) derived in Arendarczyk and Dębicki (2011).

Remarks: a) Liu and Tang (2010) considers more general Weibull-type risks and establishes under weaker conditions than ours the subexponentiality of $Z$.

b) If $K_{1}=0$ in condition (2.1), the lower bound of $\mathbb{P}\{Z>x\}$ can be substituted by

$$
\mathbb{P}\left\{X_{1}>x, X_{2}>y\right\} \geq K x^{\gamma_{1}} y^{\gamma_{2}} \mathbb{P}\left\{X_{1}>x\right\} \mathbb{P}\left\{X_{2}>y\right\}
$$

for all $x, y$ large and some constants $K>0, \gamma_{1}, \gamma_{2} \in \mathbb{R}$.

c) As can be seen from the proof of Theorem 2.1 (check in particular (4.1)), the assumption that $g_{i}, i=1,2$ is regularly varying can be slightly weakened to

$$
\lim _{\varepsilon \rightarrow 0} \lim _{u \rightarrow \infty} \frac{g_{i}((1+\varepsilon) u)}{g_{i}(u)}=1, \quad \text { and } c_{i} u^{r_{i}} \leq g_{i}(u) \leq c_{i}^{*} u^{r_{i}^{*}},
$$

where the inequalities holds for all large $u$ with constants $c_{i}>0, r_{i}, r_{i}^{*} \in \mathbb{R}, i=1,2$.

d) The constants appearing in the conditions (2.1) and (2.2) do not explicitly show in the tail asymptotics of $Z$. Our dependence model implied by the aforementioned conditions is quite restrictive. As shown below in Example 3 , complete different results are obtained if we drop some restrictions on the joint dependence of $X_{1}$ and $X_{2}$.

We present next three examples.

Example 1. Let $X_{i}, i=1,2, \cdots, m$ be Gamma distributed with scale $\lambda$ and shape $\alpha$ i.e.,

$$
\mathbb{P}\left\{X_{i}>x\right\} \sim \frac{x^{\alpha-1}}{\lambda^{\alpha-1} \Gamma(\alpha)} \exp (-x / \lambda)
$$

as $x \rightarrow \infty$. In view of (2.5), we have

$$
\mathbb{P}\left\{\prod_{i=1}^{m} X_{i}>x\right\} \sim\left(\frac{2^{m-1} \pi^{m-1}}{m \lambda^{m-1}}\right)^{\frac{1}{2}} \frac{1}{\lambda^{m \alpha-m}(\Gamma(\alpha))^{m}} x^{\frac{2 m \alpha-m-1}{2 m}} \exp \left(-\frac{m}{\lambda} x^{\frac{1}{m}}\right) .
$$

Furthermore, by (2.4), we get for the pdf $h$ of $\prod_{i=1}^{m} X_{i}$

$$
h(x) \sim \frac{x^{\frac{1}{m}-1}}{\lambda} \mathbb{P}\left\{\prod_{i=1}^{m} X_{i}>x\right\} .
$$


Example 2. Let $X_{i} \sim F_{i}, i=1,2$ be two positive random variables such that (1.1) holds and $g_{1}, g_{2}$ are ultimately monotone and regularly varying at infinity. We suppose that the joint distribution of $X_{1}$ and $X_{2}$ is FGM i.e., for $\tau \in[-1,1]$,

$$
\mathbb{P}\left\{X_{1} \leq z_{1}, X_{2} \leq z_{2}\right\}=F_{1}\left(z_{1}\right) F_{2}\left(z_{2}\right)\left(1-\tau\left(1-F_{1}\left(z_{1}\right)\right)\left(1-F_{2}\left(z_{2}\right)\right)\right)
$$

Consequently,

$$
\mathbb{P}\left\{X_{1}>x / y \mid X_{2}=y\right\}=\bar{F}_{1}(x / y)\left(1+\tau F_{1}(x / y)\left(1-2 F_{2}(y)\right)\right)
$$

where

$$
1-|\tau| \leq 1+\tau F_{1}(x / y)\left(1-2 F_{2}(y)\right)<1+|\tau|,
$$

and

$$
\mathbb{P}\left\{X_{1}>z_{1}, X_{2}>z_{2}\right\}=\bar{F}_{1}\left(z_{1}\right) \bar{F}_{2}\left(z_{2}\right)\left(1-\tau F_{1}\left(z_{1}\right) F_{2}\left(z_{2}\right)\right) \geq(1-|\tau|) \bar{F}_{1}\left(z_{1}\right) \bar{F}_{2}\left(z_{2}\right) .
$$

Hence both assumptions (2.1) and (2.6) are satisfied for FGM dependence. Further,

$$
\lim _{x \rightarrow \infty} \sup _{y \in\left[a w_{x}, a^{-1} w_{x}\right]}\left|\left(1+\tau F_{1}(x / y)\left(1-2 F_{2}(y)\right)\right)-(1-\tau)\right|=0,
$$

hence the condition (2.2) holds with $D=1-\tau$. A direct application of Theorem 2.1 yields

$$
\mathbb{P}\{Z>x\} \sim(1-\tau) A^{\frac{p_{2}}{2}}\left(\frac{2 \pi p_{2} L_{2}}{p_{1}+p_{2}}\right)^{\frac{1}{2}} x^{\frac{p_{1} p_{2}}{2\left(p_{1}+p_{2}\right)}} g_{1}\left(z_{x}^{-1} x\right) g_{2}\left(z_{x}\right) \exp \left(-B x^{\frac{p_{1} p_{2}}{p_{1}+p_{2}}}\right) .
$$

Example 3. Let $X_{i}, i=1,2$ be two standard Gaussian random variables with correlation coefficient $\rho \in(-1,1)$. For this example the dependence function is different from that of FGM treated above. In particular condition (2.1) is not satisfied since the conditional distributions are Gaussian. After some straightforward calculations we obtain

$$
\mathbb{P}\{Z>x\} \sim \frac{1+\rho}{\sqrt{2 \pi x}} \exp \left(-\frac{x}{1+\rho}\right) .
$$

Note that when $\rho=0$, then (2.9) follows directly by (1.3). The asymptotics in (2.9) shows that instead of $B$ appearing in (2.8), the term $1 /(1+\rho)$ which depends on the correlation coefficient $\rho$ appears. Our dependence structure does not imply restrictions for $B$, hence the Gaussian case is clearly not covered by the dependence model assumed in Theorem 2.1]

\section{Applications}

Our first application deals with the supremum of Brownian motion on some random interval $[0, \mathcal{T}]$. It can be easily seen that our result can be extended for several Gaussian processes using the key findings of Arendarczyk and Dębicki (2011).

Assume that $\mathcal{T}$ is almost surely positive with asymptotic tail behaviour given by (1.1) with some function $g(\cdot)$ and positive constants $L, p$. If $B(t), t \geq 0$ is a standard Brownian motion (mean 0 , variance function $t$, and continuous sample paths), then for any $x>0$, by the self-similarity property of Brownian motion we have

$$
\mathbb{P}\left\{\sup _{t \in[0, \mathcal{T}]} B(t)>x\right\}=\mathbb{P}\left\{\mathcal{T}^{1 / 2} \sup _{t \in[0,1]} B(t)>x\right\} \geq \mathbb{P}\{B(\mathcal{T})>x\}
$$


Since $\sup _{t \in[0,1]} B(t)$ has the same distribution as $|B(1)|$, if further $g(\cdot)$ is ultimately monotone, applying Corollary 2.2 we obtain

$$
\mathbb{P}\left\{\sup _{t \in[0, \mathcal{T}]} B(t)>x\right\} \sim\left(\frac{2}{1+p}\right)^{\frac{1}{2}} g\left(A x^{\frac{2}{1+p}}\right) \exp \left(-\left(\frac{1}{2 A}+L A^{p}\right) x^{\frac{2 p}{1+p}}\right),
$$

where $A=(2 L p)^{-1 /(1+p)}$. For the special simple case $g(x)=C x^{\alpha}$ with $C$ some positive constant the above claim is stated in Theorem 4.1 of Arendarczyk and Dȩbicki (2011).

Our second application is motivated by the recent paper Schlueter and Fischer (2012) which derives a formula for the weak tail dependence coefficient of elliptical generalized hyperbolic distribution (EGHD).

We shall consider below a bivariate elliptical random vector $\left(X_{1}, X_{2}\right)$ with stochastic representation

$$
\left(X_{1}, X_{2}\right) \stackrel{d}{=} R\left(U_{1}, \rho U_{1}+\sqrt{1-\rho^{2}} U_{2}\right), \quad \rho \in(-1,1),
$$

where the positive random radius $R$ is independent of $\left(U_{1}, U_{2}\right)$ which is uniformly distributed on the unit circle of $\mathbb{R}^{2}$. The basic properties of elliptical random vectors are well-known, see e.g., Cambanis et al. (1981). Assume that the random radius $R$ has distribution function $G$ in the Gumbel max-domain of attraction (see e.g., Resnick (1987)) i.e.,

$$
\lim _{x \rightarrow \infty} \frac{1-G(x+s / w(x))}{1-G(x)}=\exp (-s), \quad \forall s \in \mathbb{R}
$$

holds with some positive scaling function $w$, Hashorva (2007) obtained the exact asymptotic of tail probability of the bivariate elliptical vector

$$
\mathbb{P}\left\{X_{1}>x, X_{2}>x\right\} \sim \frac{\sqrt{c_{\rho}}}{2 \pi} \frac{\left(1-\rho^{2}\right)^{\frac{3}{2}}}{(1-\rho)^{2}} \frac{1}{x w\left(\sqrt{c_{\rho}} x\right)} \mathbb{P}\left\{R>\sqrt{c_{\rho}} x\right\},
$$

where $c_{\rho}=2 /(1+\rho)$.

For statistical modelling, calculation of the weak tail dependence coefficient is of particular interest. Hashorva (2010) derived the weak tail dependence coefficient of the elliptical distribution as

$$
\chi=2\left(\frac{1+\rho}{2}\right)^{\theta / 2}-1
$$

if

$$
\lim _{x \rightarrow \infty} \frac{w(c x)}{w(x)}=c^{\theta-1}, \quad \forall c>0
$$

holds for some $\theta \in[0, \infty)$. We extend the above results to bivariate scaled elliptical random vectors under the condition that the joint distribution of the random radius $R$ and the scaling random variable $S$ is the FGM distribution.

Theorem 3.1. Let $\left(X_{1}, X_{2}\right)$ be a bivariate elliptical random vector with representation (3.2) and define $Y_{1}=$ $S X_{1}, Y_{2}=S X_{2}$ with $S$ some positive scaling random variable. Assume that both $R$ and $S$ satisfy (1.1) with $g_{1}, g_{2}$ ultimately monotone, and have FGM distribution. If $S R$ is independent of $\left(U_{1}, U_{2}\right)$, then we have

$$
\begin{aligned}
\mathbb{P}\left\{Y_{1}>x, Y_{2}>x\right\} \sim & (1-\tau) \frac{\left(1-\rho^{2}\right)^{\frac{3}{2}}}{(1-\rho)^{2}}\left(\frac{p_{2} L_{2}}{2 \pi\left(p_{1}+p_{2}\right)}\right)^{\frac{1}{2}} c_{\rho}^{1-\frac{p_{1} p_{2}}{4\left(p_{1}+p_{2}\right)}}\left(p_{1} L_{1}\right)^{-1} A^{\frac{p_{2}}{2}+p_{1}} x^{-\frac{p_{1} p_{2}}{2\left(p_{1}+p_{2}\right)}} \\
& \times g_{1}\left(c_{\rho}^{\frac{p_{2}}{2\left(p_{1}+p_{2}\right)}} z_{x}^{-1} x\right) g_{2}\left(c_{\rho}^{\frac{p_{1}}{2\left(p_{1}+p_{2}\right)}} z_{x}\right) \exp \left(-B c_{\rho}^{\frac{p_{1} p_{2}}{2\left(p_{1}+p_{2}\right)}} x^{\frac{p_{1} p_{2}}{p_{1}+p_{2}}}\right),
\end{aligned}
$$


and the weak tail dependence coefficient of the random pair $\left(Y_{1}, Y_{2}\right)$ is given by

$$
\chi=2 \cdot\left(\frac{1+\rho}{2}\right)^{\frac{p_{1} p_{2}}{2\left(p_{1}+p_{2}\right)}}-1 .
$$

Example 4. A canonical example of a bivariate scaled elliptical distribution is the EGHD, which is now widely used in finance (see e.g., Eberlein and Keller (1995) and McNeil et al. (2005)).

Let $\left(Y_{1}, Y_{2}\right)$ be elliptical generalized hyperbolic random vector with stochastic representation

$$
\left(Y_{1}, Y_{2}\right) \stackrel{d}{=}\left(S X_{1}, S X_{2}\right)
$$

where $\left(X_{1}, X_{2}\right)$ is a bivariate Gaussian random vector with correlation coefficient $\rho$ and $N(0,1)$ components being independent of $S^{2}$ which has the generalized inverse Gaussian distribution i.e.,

$$
\begin{aligned}
\mathbb{P}\{S>x\} & \sim \frac{\left(\frac{\alpha^{2}}{\delta^{2}}\right)^{\frac{\lambda}{2}}}{2 \cdot \mathbf{K}_{\lambda}\left(\sqrt{\delta^{2} \alpha^{2}}\right)} \frac{2}{\alpha^{2}} x^{2 \lambda-2} \exp \left(-\alpha^{2} x^{2} / 2\right) \\
& =c\left(\lambda, \delta^{2}, \alpha^{2}\right) \frac{2}{\alpha^{2}} x^{2 \lambda-2} \exp \left(-\alpha^{2} x^{2} / 2\right),
\end{aligned}
$$

where $\mathbf{K}_{\lambda}$ denotes the modified Bessel function of the third kind (see Abramowitz and Stegun (1965), p. 355-494), $\alpha>0, \delta>0$ and $\lambda \in \mathbb{R}$.

Schlueter and Fischer (2012) derived the weak tail dependence coefficient of EGHD by complex calculations. Now using Theorem 3.1 we immediately obtain the tail asymptotic behaviour and weak tail dependence coefficient for EGHD risks. Indeed, if $\left(Y_{1}, Y_{2}\right)$ is an EGHD bivariate random vector defined as above, then Theorem 3.1 yields

$$
\mathbb{P}\left\{Y_{1}>x, Y_{2}>x\right\} \sim \frac{c\left(\lambda, \delta^{2}, \alpha^{2}\right)}{\sqrt{2 \pi}} \frac{(1+\rho)^{3 / 2}}{(1-\rho)^{1 / 2}} \alpha^{-\lambda-\frac{3}{2}} c_{\rho}^{\frac{2 \lambda+1}{4}} x^{\frac{2 \lambda-3}{2}} \exp \left(-\alpha \sqrt{c_{\rho}} x\right)
$$

and the weak tail dependence coefficient is

$$
\chi=2\left(\frac{1+\rho}{2}\right)^{\frac{1}{2}}-1
$$

Note that (3.4) is claimed (but the formula there is not correct) in Theorem 3 of the aforementioned paper.

\section{Proofs}

Proof of Theorem 2.1 First by $(2.1)$ we have $\left(\right.$ recall $\left.w_{x}=x^{\frac{p_{1}}{p_{1}+p_{2}}}\right)$

$$
\begin{aligned}
\mathbb{P}\{Z>x\}=\bar{H}(x) & =\int_{0}^{\infty} c(x, y) \bar{F}_{1}\left(\frac{x}{y}\right) \mathrm{d} F_{2}(y) \\
& \geq \int_{w_{x}}^{\infty} c(x, y) \bar{F}_{1}\left(\frac{x}{y}\right) \mathrm{d} F_{2}(y) \\
& \geq K_{1} \int_{w_{x}}^{\infty} x^{\beta_{1}} \bar{F}_{1}\left(\frac{x}{y}\right) \mathrm{d} F_{2}(y) \\
& \geq K_{1} x^{\beta_{1}} \bar{F}_{1}\left(x w_{x}^{-1}\right) \bar{F}_{2}\left(w_{x}\right) .
\end{aligned}
$$

By the assumptions for some small $a_{1}>0$ we obtain

$$
\int_{0}^{a_{1} w_{x}} \bar{F}_{1}\left(\frac{x}{y}\right) c(x, y) \mathrm{d} F_{2}(y) \leq K_{2} x^{\beta_{2}} \int_{0}^{a_{1} w_{x}} \bar{F}_{1}\left(\frac{x}{y}\right) \mathrm{d} F_{2}(y)
$$




$$
\leq \quad K_{2} x^{\beta_{2}} \bar{F}_{1}\left(a_{1}^{-1} x w_{x}^{-1}\right)=o(\bar{H}(x))
$$

Similarly, for some large $a_{2}>0$ we obtain

$$
\int_{a_{2} w_{x}}^{\infty} \bar{F}_{1}\left(\frac{x}{y}\right) c(x, y) \mathrm{d} F_{2}(y) \leq K_{2} x^{\beta_{2}} \bar{F}_{2}\left(a_{2} w_{x}\right)=o(\bar{H}(x)) .
$$

Next, by Lemma A.5 in Tang and Tsitsiashvili (2004) we can assume that without loss of generality that $F_{2}$ is absolutely continuous and therefore we take simply $F_{2}(x)=1-g_{2}(x) \exp \left(-L_{2} x^{p_{2}}\right), x>0$. Further, in view of Theorem 1.3.3 of Bingham et al. (1987) we can assume that $g_{2}$ is a normalised slowly varying function with derivative $g_{2}^{\prime}$. Consequently, for all large $x$

$$
\begin{aligned}
\mathbb{P}\{Z>x\} & \sim \int_{a_{1} w_{x}}^{a_{2} w_{x}} \bar{F}_{1}(x / y) c(x, y) \mathrm{d} F_{2}(y) \\
& \sim-D x^{q_{1}} \int_{a_{1} w_{x}}^{a_{2} w_{x}} y^{q_{2}-q_{1}} \bar{F}_{1}(x / y) \mathrm{d}\left(g_{2}(y) \exp \left(-L_{2} y^{p_{2}}\right)\right) \\
& =D x^{q_{1}} L_{2} p_{2} \int_{a_{1} w_{x}}^{a_{2} w_{x}} y^{q_{2}-q_{1}+p_{2}-1} \bar{F}_{1}(x / y) g_{2}(y) \exp \left(-L_{2} y^{p_{2}}\right)\left[1-\frac{g_{2}^{\prime}(y)}{g_{2}(y) L_{2} p_{2}} y^{1-p_{2}}\right] \mathrm{d} y \\
& \sim D L_{2} p_{2} x^{q_{1}} \int_{a_{1} w_{x}}^{a_{2} w_{x}} y^{q_{2}-q_{1}+p_{2}-1} g_{1}(x / y) g_{2}(y) \exp \left(-L_{1}(x / y)^{p_{1}}-L_{2} y^{p_{2}}\right) \mathrm{d} y .
\end{aligned}
$$

We write further

$I_{1}(x)+I_{2}(x)+I_{3}(x)=\left(\int_{a_{1} w_{x}}^{(1+\varepsilon)^{-\frac{1}{p_{2}}} z_{x}}+\int_{(1+\varepsilon)^{-\frac{1}{p_{2}}} z_{x}}^{(1+\varepsilon)^{\frac{1}{p_{2}}} z_{x}}+\int_{(1+\varepsilon)^{\frac{1}{p_{2}}} z_{x}}^{a_{2} w_{x}}\right) y^{q_{2}-q_{1}+p_{2}-1} g_{1}(x / y) g_{2}(y) \exp \left(-L_{1}(x / y)^{p_{1}}-L_{2} y^{p_{2}}\right) \mathrm{d} y$,

where $\varepsilon>0, z_{x}=A w_{x}$ and $A$ is given by (1.4). Note that the function $\psi(y)=L_{1}(x / y)^{p_{1}}+L_{2} y^{p_{2}}$ decreases when $0<y \leq z_{x}$ and increases when $y \geq z_{x}$. As in Liu and Tang (2010), we obtain

$$
I_{1}(x) \leq \exp \left(-\left((1+\varepsilon)^{\frac{p_{1}}{p_{2}}} L_{1} A^{-p_{1}}+(1+\varepsilon)^{-1} L_{2} A^{p_{2}}\right) x^{\frac{p_{1} p_{2}}{p_{1}+p_{2}}}\right) \int_{a_{1} w_{x}}^{(1+\varepsilon)^{-\frac{1}{p_{2}}} z_{x}} g_{1}\left(\frac{x}{y}\right) g_{2}(y) y^{q_{2}-q_{1}+p_{2}-1} \mathrm{~d} y
$$

and

$$
I_{3}(x) \leq \exp \left(-\left((1+\varepsilon)^{-\frac{p_{1}}{p_{2}}} L_{1} A^{-p_{1}}+(1+\varepsilon) L_{2} A^{p_{2}}\right) x^{\frac{p_{1} p_{2}}{p_{1}+p_{2}}}\right) \int_{(1+\varepsilon)^{\frac{1}{p_{2}}} z_{x}}^{a_{2} w_{x}} g_{1}\left(\frac{x}{y}\right) g_{2}(y) y^{q_{2}-q_{1}+p_{2}-1} \mathrm{~d} y .
$$

Next, we have

$$
\begin{aligned}
& I_{2}(x) \\
\geq & \left(\int_{(1+\varepsilon / 2)^{-\frac{1}{p_{2}}} z_{x}}^{z_{x}}+\int_{z_{x}}^{(1+\varepsilon / 2)^{\frac{1}{p_{2}}} z_{x}}\right) y^{q_{2}-q_{1}+p_{2}-1} g_{1}\left(\frac{x}{y}\right) g_{2}(y) \exp \left(-\left(L_{1}\left(\frac{x}{y}\right)^{p_{1}}+L_{2} y^{p_{2}}\right)\right) \mathrm{d} y \\
\geq & \exp \left(-\left((1+\varepsilon / 2)^{\frac{p_{1}}{p_{2}}} L_{1} A^{-p_{1}}+(1+\varepsilon / 2)^{-1} L_{2} A^{p_{2}}\right) x^{\frac{p_{1} p_{2}}{p_{1}+p_{2}}}\right) \int_{(1+\varepsilon / 2)^{-\frac{1}{p_{2}}} z_{x}}^{z_{x}} y^{q_{2}-q_{1}+p_{2}-1} g_{1}\left(\frac{x}{y}\right) g_{2}(y) \mathrm{d} y \\
& +\exp \left(-\left((1+\varepsilon / 2)^{-\frac{p_{1}}{p_{2}}} L_{1} A^{-p_{1}}+(1+\varepsilon / 2) L_{2} A^{p_{2}}\right) x^{\frac{p_{1} p_{2}}{p_{1}+p_{2}}}\right) \int_{z_{x}}^{(1+\varepsilon / 2)^{\frac{1}{p_{2}}} z_{x}} y^{q_{2}-q_{1}+p_{2}-1} g_{1}\left(\frac{x}{y}\right) g_{2}(y) \mathrm{d} y .
\end{aligned}
$$

Consequently, as in Liu and Tang (2010), using Taylor's expansion we obtain $I_{1}(x)=o\left(I_{2}(x)\right)$ and $I_{3}(x)=o\left(I_{2}(x)\right)$, implying thus for all $\varepsilon>0$

$$
\bar{H}(x) \sim D L_{2} p_{2} x^{q_{1}} I_{2}(x)=D L_{2} p_{2} x^{q_{1}} \int_{(1+\varepsilon)^{-\frac{1}{p_{2}}} z_{x}}^{(1+\varepsilon)^{\frac{1}{p_{2}}} z_{x}} g_{1}\left(\frac{x}{y}\right) g_{2}(y) y^{q_{2}-q_{1}+p_{2}-1} \exp \left(-L_{1}\left(\frac{x}{y}\right)^{p_{1}}-L_{2} y^{p_{2}}\right) \mathrm{d} y .
$$


Since $g_{1}(\cdot), g_{2}(\cdot)$ are ultimately monotone, assume without loss of generality that they are both ultimately increasing. Hence for $y \in\left[(1+\varepsilon)^{-\frac{1}{p_{2}}} z_{x},(1+\varepsilon)^{\frac{1}{p_{2}}} z_{x}\right]$ we have

$$
g_{1}\left((1+\varepsilon)^{-\frac{1}{p_{2}}} z_{x}^{-1} x\right) g_{2}\left((1+\varepsilon)^{-\frac{1}{p_{2}}} z_{x}\right) \leq g_{1}\left(\frac{x}{y}\right) g_{2}(y) \leq g_{1}\left((1+\varepsilon)^{\frac{1}{p_{2}}} z_{x}^{-1} x\right) g_{2}\left((1+\varepsilon)^{\frac{1}{p_{2}}} z_{x}\right) \text {. }
$$

By letting $\varepsilon \rightarrow 0$ and using the Laplace approximation we obtain

$\bar{H}(x) \sim D L_{2} p_{2} x^{q_{1}} I_{2}(x) \sim D \sqrt{\frac{2 \pi p_{2} L_{2}}{p_{1}+p_{2}}} A^{\frac{p_{2}}{2}+q_{2}-q_{1}} x^{\frac{2 p_{1} q_{2}+2 p_{2} q_{1}+p_{1} p_{2}}{2\left(p_{1}+p_{2}\right)}} g_{1}\left(z_{x}^{-1} x\right) g_{2}\left(z_{x}\right) \exp \left(-\left(L_{1} A^{-p_{1}}+L_{2} A^{p_{2}}\right) w_{x}^{p_{2}}\right)$,

and thus the proof is complete.

Proof of Corollary 2.2 The tail asymptotic of the distribution of $Z$ follows easily, therefore we show next the tail asymptotic of the pdf $h$ of $Z$. For all $x$ large and $\epsilon \in(0,1)$, since $h_{1}$ is ultimately decreasing

$$
\begin{aligned}
h(x)= & \int_{0}^{\infty} h_{1}\left(\frac{x}{y}\right) \frac{1}{y} \mathrm{~d} F_{2}(y) \\
\geq & \int_{w_{x}}^{2 w_{x}} h_{1}\left(\frac{x}{y}\right) \frac{1}{y} \mathrm{~d} F_{2}(y) \\
\geq & 2^{-1} w_{x}^{-1} h_{1}\left(x w_{x}^{-1}\right)\left[\bar{F}_{2}\left(w_{x}\right)-\bar{F}_{2}\left(2 w_{x}\right)\right] \\
\geq & (1-\epsilon) 2^{-1} L_{1} p_{1} x^{p_{1}-\frac{p_{1}^{2}}{p_{1}+p_{2}}-1} g_{1}\left(x w_{x}^{-1}\right) g_{2}\left(w_{x}\right) \exp \left(-\left(L_{1}+L_{2}\right) w_{x}^{p_{2}}\right) \\
& \times\left(1-\frac{g_{2}\left(2 w_{x}\right)}{g_{2}\left(w_{x}\right)} \exp \left(-L_{2}\left(2^{p_{2}}-1\right) w_{x}^{p_{2}}\right)\right) .
\end{aligned}
$$

Let $X^{*}$ be a positive random variable with distribution function $F^{*}$ which satisfies

$$
\bar{F}^{*}(x) \sim x^{p_{1}} g_{1}(x) \exp \left(-L_{1} x^{p_{1}}\right)
$$

For some $a_{1}>0$ small enough we have

$$
\begin{aligned}
\int_{0}^{a_{1} w_{x}} h_{1}\left(\frac{x}{y}\right) \frac{1}{y} \mathrm{~d} F_{2}(y) & \leq(1+\epsilon) L_{1} p_{1} x^{-1} \int_{0}^{a_{1} w_{x}} \bar{F}^{*}\left(\frac{x}{y}\right) \mathrm{d} F_{2}(y) \\
& \leq(1+\epsilon) L_{1} p_{1} x^{-1} \bar{F}^{*}\left(a_{1}^{-1} w_{x}^{-1} x\right)=o(h(x)) .
\end{aligned}
$$

Similarly, for some large $a_{2}>0$, since $h_{1}$ is bounded, there exists a positive constant $M$ such that

$$
\int_{a_{2} w_{x}}^{\infty} h_{1}\left(\frac{x}{y}\right) \frac{1}{y} \mathrm{~d} F_{2}(y) \leq M a_{2}^{-1} w_{x}^{-1} g_{2}\left(a_{2} w_{x}\right) \exp \left(-L_{2} a_{2}^{p_{2}} w_{x}^{p_{2}}\right)=o(h(x)) .
$$

Consequently, with the same arguments as in the proof of Theorem 2.1 we obtain

$$
\begin{aligned}
h(x) & \sim \int_{a_{1} w_{x}}^{a_{2} w_{x}} h_{1}\left(\frac{x}{y}\right) \frac{1}{y} \mathrm{~d} F_{2}(y) \\
& \sim L_{1} L_{2} p_{1} p_{2} x^{p_{1}-1} \int_{a_{1} w_{x}}^{a_{2} w_{x}} y^{p_{2}-p_{1}-1} g_{1}\left(\frac{x}{y}\right) g_{2}(y) \exp \left(-L_{1}\left(\frac{x}{y}\right)^{p_{1}}-L_{2} y^{p_{2}}\right) \mathrm{d} y
\end{aligned}
$$

hence the proof follows easily.

Proof of Theorem 3.1 In view of 3.3

$$
\begin{aligned}
\mathbb{P}\left\{S X_{1}>x, S X_{2}>x\right\} \sim & \frac{1}{2 \pi} \frac{\left(1-\rho^{2}\right)^{\frac{3}{2}}}{(1-\rho)^{2}} \frac{p_{1}+p_{2}}{p_{1} p_{2}}\left(L_{1} A^{-p_{1}}+L_{2} A^{p_{2}}\right)^{-1} \\
& \times\left(\frac{2}{1+\rho}\right)^{-\frac{p_{1} p_{2}}{2\left(p_{1}+p_{2}\right)}+1} x^{-\frac{p_{1} p_{2}}{p_{1}+p_{2}}} \mathbb{P}\left\{S R>\sqrt{\frac{2}{1+\rho}} x\right\}
\end{aligned}
$$


and thus by (2.8) the first claim follows. Since $Z$ is in the Gumbel max-domain of attraction with scaling function $w(\cdot)$ given by

$$
w(x)=\left(L_{1} A^{-p_{1}}+L_{2} A^{p_{2}}\right) \frac{p_{1} p_{2}}{p_{1}+p_{2}} x^{\frac{p_{1} p_{2}}{p_{1}+p_{2}}-1},
$$

then by Theorem 2.1 of Hashorva (2010) for any $c>0$ we have

$$
\lim _{x \rightarrow \infty} \frac{w(c x)}{w(x)}=c^{\frac{p_{1} p_{2}}{p_{1}+p_{2}}-1}
$$

hence the weak tail dependence coefficient is

$$
\chi=2 \cdot\left(\frac{1+\rho}{2}\right)^{\frac{p_{1} p_{2}}{2\left(p_{1}+p_{2}\right)}}-1
$$

establishing thus the proof.

Acknowledgments: We would like to thank both referees for constructive suggestions that improved our paper significantly. Z. Weng kindly acknowledges the full financial support by the Swiss National Science Foundation Grant 200021-134785.

\section{References}

[1] Abramowitz, M. and Stegun, I.A. (1965). Handbook of Mathematical Functions. Dover, New York.

[2] Arendarczyk, M. and Dȩbicki, K. (2011). Asymptotics of supremum distribution of a Gausssian process over a Weibullian time. Bernoulli. 17, 194-210.

[3] Arendarczyk, M. and Dȩbicki, K. (2012). Exact asymptotics of supremum of a stationary Gaussian process over a random interval. Stat. Probab. Lett. 82, 645-652.

[4] Balakrishnan, N. and Hashorva E. 2011. On Kotz-Pearson Dirichlet distributions. J. Multivariate Anal., 102, 948-957.

[5] Berman, M.S. (1992). Sojourns and Extremes of Stochastic Processes. Wadsworth \& Brooks/Cole.

[6] Bingham, N., Goldie, C.M. and Teugels, J.L. (1987). Regular Variations. Cambridge, Cambridge University Press.

[7] Bose, A., Hazra, R.S. and Saha, K. (2012). Product of exponentials and spectral radius of random k circulants. Annales de l'Institut Henri Poincare Probabilites et Statistiques. 48, 424-443.

[8] Cambanis, S., Huang, S. and Simons, G. (1981). On the theory of elliptically contoured distributions. J. Multivariate Analysis. 11, 368-385.

[9] Chen, Y. (2011). The finite-time ruin probability with dependent insurance and financial risks. J. Appl. Probab. 48, 1035-1048.

[10] Cline, D.B.H. and Samorodnitsky, G. (1994). Subexponentiality of the product of independent random variables. Stochast. Process. Appl. 49, 75-98. 
[11] Constantinescu, C., Hashorva, E. and Ji, L. (2011). The Archimedean copula in finite and infinite dimensions - with applications to ruin problems. Insurance: Mathem and Econom. 49, 487-495.

[12] Eberlein, E. and Keller, U. (1995). Hyperbolic distributions in finance. Bernoulli. 1, 281-299.

[13] Hashorva, E. (2007). Asymptotics properties of Type I elliptical random vectors. Extremes. 10, 175-206.

[14] Hashorva, E. (2010). On the residual dependence index of elliptical distributions. Stat. Probab. Lett. 80, 1070-1078.

[15] Hashorva, E. (2011). On beta-product convolutions. Scandinavian Actuarial J. doi:10.1080/03461238.2011.555939.

[16] Hashorva, E. (2012). Exact tail asymptotics in bivariate scale mixture models. Extremes. 15, 109-128.

[17] Hashorva, E., Ji, L. and Tan, Z. (2012). On the infinite sums of deflated Gaussian products. Elect. Comm. Probab. 17, 1-8.

[18] Hashorva, E. and Pakes, A.G. (2010). Tail asymptotics under beta random scaling. J. Math. Anal. Appl. 372, $496-514$.

[19] Hashorva, E., Pakes, A.G. and Tang, Q. (2010). Asymptotics of random contractions. Insurance: Mathematics and Economics 47, 405-414.

[20] Jessen, A.H. and Mikosch, T. (2006). Regularly varying functions. Publications de l'Institut Mathmatique, Nouvelle Série, 80, 171-192.

[21] Jiang, J. and Tang, Q. (2011). The product of two dependent random variables with regularly varying or rapidly varying tails. Stat. Probab. Lett. 81, 957-961.

[22] Liu, Y. and Tang, Q. (2010). The subexponential product convolution of two Weibull-type distributions. J. Aust. Math. Soc. 89, 277-288.

[23] Maulik, K. and Resnick, S. (2004). Characterizations and examples of hidden regular variation. Extremes. 7, $31-67$.

[24] McNeil, A. J., Frey, R. and Embrechts, P. (2005). Quantitative risk management: concepts, techniques, and tools. Princeton University Press, Princeton.

[25] Nadarajah, S. (2005). Sums, products and ratios of generalized beta variables. Stat. Papers. 47, 69-90.

[26] Nadarajah, S. and Kotz, S. (2005). On the product and ratio of gamma and beta random variables. Allgemeines Statistisches Archiv. 89, 435-449.

[27] Pakes, A.G. and Navarro, J. (2007). Distributional characterizations through scaling relations. Aust. \& N.Z. J. Stat. 49, 115-135.

[28] Resnick, S.I. (1987). Extreme Values, Regular Variation and Point Processes. Springer, New York. 
[29] Schlueter, S. and Fischer, M. (2012). The weak tail dependence coefficient of the elliptical generalized hyperbolic distriubtion. Extremes. 15, 159-174.

[30] Tang, Q. and Tsitsiashvili, G. (2004). Finite- and infinite-time ruin probabilities in the presence of stochastic returns on investments. Adv. Appl. Probab. 36, 1278-1299.

[31] Tang, Q. (2006a). On convolution equivalence with applications. Bernoulli. 12, 535-549.

[32] Tang, Q. (2006b). The subexponentiality of products revisited. Extremes. 9, 231-241.

[33] Tang, Q. (2008). From light tails to heavy tails through multiplier. Extremes. 11, 379-391.

[34] Yang, Y. and Wang, Y. (2012). Tail behavior of the product of two dependent random variables with applications to risk theory. Extremes. doi:10.1007/s10687-012-0153-2.

[35] Yang, Y., Hu, S. and Wu, T. (2011). The tail probability of the product of dependent random variables from max-domains of attraction. Stat. Probab. Lett. 81, 1876-1882.

[36] Zwart, B., Borst, S. and Debicki, K. (2005). Subexponential asymptotics of hybrid fluid and ruin models. Ann. Appl. Probab. 15, 500-517. 